Quantum Information and Computation, Vol. 9, No. 7\&8 (2009) 0657-0665

(C) Rinton Press

\title{
CORRELATION LOSS AND MULTIPARTITE ENTANGLEMENT ACROSS A BLACK HOLE HORIZON
}

\author{
GERARDO ADESSO \\ School of Mathematical Sciences, University of Nottingham \\ University Park, Nottingham NG7 2RD, UK. \\ IVETTE FUENTES-SCHULLER ${ }^{a}$ \\ Institute for Theoretical Physics, Technical University of Berlin \\ Hardenbergstr. 36, D-10623, Berlin, Germany \\ Quantum Physics Group, STRI, School of Physics, Astronomy and Mathematics \\ University of Hertfordshire, Hatfield, Herts AL10 9AB, United Kingdom \\ Received September 16, 2008 \\ Revised March 20, 2009
}

\begin{abstract}
We investigate the Hawking effect on entangled fields. By considering a scalar field which is in a two-mode squeezed state from the point of view of freely falling (Kruskal) observers crossing the horizon of a Schwarzschild black hole, we study the degradation of quantum and classical correlations in the state from the perspective of Schwarzschild observers confined outside the horizon. Due to monogamy constraints on the entanglement distribution, we show that the lost bipartite entanglement is recovered as multipartite entanglement among modes inside and outside the horizon. In the limit of a small-mass black hole, no bipartite entanglement is detected outside the horizon, while the genuine multipartite entanglement interlinking the inner and outer regions grows infinitely.

Keywords: Multipartite entanglement, continuous variable correlations, Hawking-Unruh effect, Schwarzschild black holes

Communicated by: S Braunstein \& E Polzik
\end{abstract}

\section{Introduction}

The physics of black holes has attracted and fascinated scientists since almost eighty years. Recently, in the quest to understand and properly describe such singular objects, new insights have been drawn into and from apparently unrelated areas, such as condensed matter physics and information theory. On the one hand, inspired by the holographic principle, the investigation of the "area law" entropy scaling in Hamiltonian lattice systems has unveiled general connections between decay of correlations, spectral gap, and computational complexity of ground states [1]. On the other hand, the concepts of entropy, information, and entanglement have been developed into the novel field of quantum information science, whose applications are revolutionizing modern communications 2. It seems interesting to investigate whether the novel tools developed in these areas may prove useful, in turn, to achieve a better comprehension of traditional black hole questions [3].

${ }^{a}$ Published before under maiden name Fuentes-Guridi. 
In particular, quantum entanglement has been recognized to play an important role in black hole thermodynamics [4] and in the information loss problem in a black hole [5], one of the most challenging issues in theoretical physics at the moment [6]. Consider a snapshot of the gravitational collapse of a massive object forming a Schwarzschild black hole. A scalar field which is in the so-called Unruh vacuum state [7 from the point of view of freely falling observers at the horizon (Kruskal observers) [8], contains quantum correlations between the field modes inside and outside the horizon. Namely, the modes described by observers living outside the black hole (outer Schwarzschild observers) are entangled with the modes described by virtual observers confined to the interior of the black hole (inner Schwarzschild observers) [9. Such entanglement is directly related to the entropy of the black hole [4]. Schwarzschild observers outside the black hole have no access to the modes inside the horizon, thus have incomplete information about the quantum field, which results in the detection of a thermal state. The so-called Hawking temperature of such a state is inversely proportional to the black hole mass $M$ [7]. For a black hole with small, asymptotically vanishing mass (corresponding e.g. to a snapshot taken close to the final evaporation stage), the radiation temperature approaches infinity, resulting in the detection of a maximally mixed state which contains no information. This is the core of the 'paradox' [6]: according to Hawking's analysis, a black hole can "swallow" the information about pure quantum states and eventually disappear alongwith it.

Here we face an intriguing question: what if an entangled state of the field is detected by infalling observers? Will the black hole "swallow" also the information encoded in correlations between the field modes? We provide answers to these and related questions in the following. Let us remark that in the canonical studies of the Hawking radiation [9], the state of the scalar field, from the perspective of free falling observers, is the vacuum state. Since the vacuum state contains no entanglement from this perspective, it is not possible to address the question of how much quantum information (encoded in correlations between field modes) is lost from the perspective of physical observers living outside the black hole. However, in principle, and quite realistically, the field from the Kruskal perspective could be in any state. The most general case would correspond to multiparty entangled states of an arbitrary number $N$ of field modes. Unfortunately, the entanglement content of general multipartite states cannot be exactly quantified with the existing entanglement measures, and even its qualification is a quite formidable task. In our analysis we take the first step beyond the conventional studies by considering a scalar field in a $1+1$ dimensional Schwarzschild black hole spacetime, which is in a two-mode squeezed entangled state from the point of view of (freely falling) Kruskal observers. This represents one of the simplest nontrivial instances of $N$ entangled modes (being, in fact, $N=2$ ). Importantly, for the studied case analytical tools have been developed to quantify bipartite entanglement and its redistribution into multipartite form when the field is observed from a different perspective [12. Therefore our analysis generalizes the standard studies where the tools of quantum information theory were applied to black hole physics [4, 5, 6. The canonical studies are actually recovered when the entanglement between the two modes vanishes from the Kruskal perspective, in which case two independent Kruskal vacuum field modes are detected as equally thermalized states by Schwarzschild observers. But the most interesting contribution of our study is that considering the two-mode squeezed state enables us to address novel questions such as what is the effect of a black hole on the 
correlations (quantum and classical) present in a multimode field. The specific choice of a twomode squeezed state as entangled state of the field as detected by Kruskal observers is very natural, also in view of the ubiquity of two-mode squeezed states in black hole physics (both in connection to its entropy [4, and to its final state [10]) [13. Moreover, in curved spacetimes with at least two asymptotically flat regions, particle states [11 commonly correspond to multiparty squeezed entangled states of an arbitrary number $N$ of field modes. Such is the case, as an example, in a Robertson-Walker Universe. Our setting deals with the paradigmatic case $N=2$.

We show analytically that the Kruskal correlations, both quantum and classical, in the state, are smaller when detected by Schwarzschild observers outside the horizon; namely, they are more intensively degraded for smaller black hole mass $M$ and lower mode frequencies. In the limit $M \rightarrow 0$, quantum correlations vanish for all frequency modes, but some classical correlations remain in the state, although smaller than those detected by Kruskal observers. An original insight is provided for the Hawking mechanism: we demonstrate that the entanglement loss is precisely reciprocated by a redistribution of the Kruskal bipartite entanglement into genuine multipartite quantum correlations between Schwarzschild modes inside and outside the black hole. In the limit $M \rightarrow 0$, the detection of an even finite bipartite entanglement by infalling observers, amounts to the creation of an unbounded multipartite entanglement across the horizon. Our results thus shed new light on the nature of the information lost in black holes, within the quantum-informational framework of monogamy of entanglement [15].

\section{The setting}

Let us briefly recall the Hawking effect on a scalar field. Each Unruh vacuum mode of frequency $\alpha$, denoted by $|0\rangle_{\alpha}^{\mathcal{K}}$ in the Kruskal frame, corresponds to a two-mode squeezed state in the Schwarzschild frame [8, 7, 9] (see also Ref. [10]),

$$
\begin{aligned}
|0\rangle_{\alpha}^{\mathcal{K}} & =\frac{1}{\cosh r} \sum_{k=0}^{\infty} \tanh ^{k} r|k\rangle_{\alpha}^{\text {in }}|k\rangle_{\alpha}^{\text {out }} \\
& \equiv U_{\alpha^{\text {in }, \alpha^{\text {out }}}(r)|0\rangle_{\alpha}^{\text {in }}|0\rangle_{\alpha}^{\text {out }} .}
\end{aligned}
$$

Here $|k\rangle_{\alpha}^{\text {in }}$ and $|k\rangle_{\alpha}^{\text {out }}$ are the Fock states inside and outside the black hole, $U_{i, j}(r)=\exp \frac{r}{2}\left(\hat{a}_{i}^{\dagger} \hat{a}_{j}^{\dagger}-\right.$ $\hat{a}_{i} \hat{a}_{j}$ ) is the two-mode squeezing operator with $r$ the (real) squeezing parameter, and $a_{i, j}, a_{i, j}^{\dagger}$, are creation and annihilation operators for modes $i, j$. The squeezing $r$ is related to the frequency $\alpha$ and the mass $M$ of the black hole (in natural units),

$$
\cosh r=\left(1-e^{-2 M \pi \alpha}\right)^{-1 / 2} .
$$

The two-mode squeezed state of Eq. (1) is a Gaussian state, i.e. a continuous variable state with Gaussian characteristic function [12. Up to local unitary operations, all the information about a Gaussian state is contained in the symmetric covariance matrix $(\mathrm{CM})$ whose entries are $\sigma_{i j}=1 / 2\left\langle\left\{\hat{X}_{i}, \hat{X}_{j}\right\}\right\rangle-\left\langle\hat{X}_{i}\right\rangle\left\langle\hat{X}_{j}\right\rangle$ where $\hat{X}=\left\{\hat{x}_{1}, \hat{p}_{1}, \ldots, \hat{x}_{N}, \hat{p}_{N}\right\}$ is the vector of the field quadrature operators. In terms of CMs, Eq. (11) reads $\boldsymbol{\sigma}_{\alpha}^{(0) \mathcal{K}}=\Gamma_{\alpha^{\text {in }}, \alpha^{\text {out }}}(r)\left(\boldsymbol{\sigma}_{\alpha}^{(0) \text { in }} \oplus\right.$ $\left.\boldsymbol{\sigma}_{\alpha}^{(0) o u t}\right) \Gamma_{\alpha^{\text {in }}, \alpha^{\text {out }}}^{T}(r)$, where $\boldsymbol{\sigma}_{\alpha}^{(0) \text { in }}=\boldsymbol{\sigma}_{\alpha}^{(0) \text { out }}=\mathbb{I}_{2}$ and $\Gamma_{i, j}(r)$ is the symplectic transformation 
associated to the two-mode squeezing operator $U_{i, j}(r)$,

$$
\Gamma_{i, j}(r)=\left(\begin{array}{cc}
\cosh (r) \mathbb{I}_{2} & \sinh (r) Z_{2} \\
\sinh (r) Z_{2} & \cosh (r) \mathbb{I}_{2}
\end{array}\right), \text { with } Z_{2}=\left(\begin{array}{cc}
1 & 0 \\
0 & -1
\end{array}\right)
$$

Suppose now to have, from the perspective of Kruskal observers, an entangled two-mode squeezed state, which is described by the $\mathrm{CM} \boldsymbol{\sigma}_{\lambda \nu}^{\mathcal{K}}(\xi)=\Gamma_{\lambda \nu}(\xi) \Gamma_{\lambda \nu}^{T}(\xi), \lambda$ and $\nu$ being the frequencies of the modes. The entropy of entanglement [16] between the modes $\lambda$ and $\nu$ is $S_{\xi}=f(\cosh 2 \xi)$, where

$$
f(x) \equiv \frac{x+1}{2} \log \left[\frac{x+1}{2}\right]-\left(\frac{x-1}{2}\right) \log \left[\frac{x-1}{2}\right] .
$$

The two modes also share classical correlations, equal in content to the entanglement $S_{\xi}$. This is because the mutual information [17, quantifying the total (quantum plus classical) amount of correlations in the state, is equal to $I_{\xi}=2 S_{\xi}$. In the limit of infinite squeezing $(\xi \rightarrow \infty)$, the state approaches the ideal (unnormalizable) EPR state [18, which contains an infinite amount of entanglement and mean energy.

We now wish to describe the state from the perspective of Schwarzschild observers which have only access to the field outside the black hole horizon. Each Kruskal mode is thus transformed according to the Bogoliubov transformation which acts on the vacuum as in Eq. (11) 7, 9] (see also [14). The global state, involving modes inside and outside the black hole, will be thus a four-mode pure Gaussian state with $\mathrm{CM} \sigma_{\lambda \nu}^{i n, o u t}=O O^{T}$, where $O=$ $\Gamma_{\nu^{\text {in }}, \nu^{\text {out }}}(n) \Gamma_{\lambda^{\text {in }}, \lambda^{\text {out }}}(l) \Gamma_{\lambda \nu}(\xi)$, and the parameters $l$ and $n$ are related to the black hole mass $M$ and to the mode frequencies $\lambda, \nu$ respectively, by Eq. (2). Explicitly,

$$
\sigma_{\lambda \nu}^{\text {in }, \text { out }}=\left(\begin{array}{cccc}
\sigma_{\lambda^{\text {in }}} & \varepsilon_{\lambda^{\text {in }} \lambda^{\text {out }}} & \varepsilon_{\lambda^{\text {in }} \nu^{\text {out }}} & \varepsilon_{\lambda^{\text {in }} \nu^{\text {in }}} \\
\varepsilon_{\lambda^{\text {in }} \lambda^{\text {out }}}^{\text {to }} & \sigma_{\lambda^{\text {out }}} & \varepsilon_{\lambda^{\text {out }} \nu^{\text {out }}} & \varepsilon_{\lambda^{\text {out }} \nu^{\text {in }}} \\
\varepsilon_{\lambda^{\text {in }} \nu^{\text {out }}}^{T} & \varepsilon_{\lambda^{\text {out }} \nu^{\text {out }}}^{T} & \sigma_{\nu^{\text {out }}} & \varepsilon_{\nu^{\text {out }} \nu^{\text {in }}} \\
\varepsilon_{\lambda^{\text {in }} \nu^{\text {in }}}^{T} & \varepsilon_{\lambda^{\text {out }} \nu^{\text {in }}}^{T} & \varepsilon_{\nu^{\text {out }} \nu^{\text {in }}}^{T} & \sigma_{\nu^{\text {in }}}
\end{array}\right),
$$

where (setting $\mathrm{c}=\cosh$ and $\mathrm{s}=\sinh$ ):

$$
\begin{aligned}
& \boldsymbol{\sigma}_{X^{i n}}=\left[\mathrm{c}^{2}(x)+\mathrm{c}(2 \xi) \mathrm{s}^{2}(x)\right] \mathbb{I}_{2}, \\
& \boldsymbol{\sigma}_{X^{\text {out }}}=\left[\mathrm{c}^{2}(x) \mathrm{c}(2 \xi)+\mathrm{s}^{2}(x)\right] \mathbb{I}_{2}, \\
& \varepsilon_{X^{\text {in }} X^{\text {out }}}=\varepsilon_{X^{\text {out }} X^{\text {in }}}=\left[\mathrm{c}^{2}(\xi) \mathrm{s}(2 x)\right] Z_{2}, \\
& \varepsilon_{X^{\text {in }} Y^{\text {out }}}=\varepsilon_{Y^{\text {out }} X^{\text {in }}}=[\mathrm{c}(y) \mathrm{s}(2 \xi) \mathrm{s}(x)] \mathbb{I}_{2} \text {, } \\
& \varepsilon_{X^{i n} Y^{i n}}=[\mathrm{s}(2 \xi) \mathrm{s}(x) \mathrm{s}(y)] Z_{2}, \\
& \varepsilon_{X^{\text {out }} Y^{\text {out }}}=[\mathrm{c}(x) \mathrm{c}(y) \mathrm{s}(2 \xi)] Z_{2} .
\end{aligned}
$$

Here $X, Y=\{\lambda, \nu\}$ and accordingly $x, y=\{l, n\}$.

\section{Bipartite entanglement and correlations between the outer modes}

The observers moving outside the black hole have access only to modes $\lambda^{\text {out }}$ and $\nu^{\text {out }}$. Therefore, after tracing over the states in the region inside the black hole, the resulting state is 

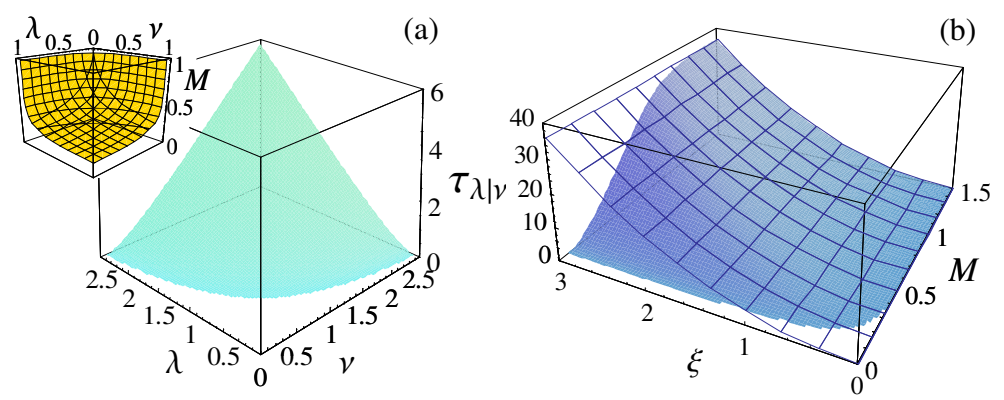

Fig. 1. Entanglement between two Schwarzschild modes detected outside a black hole (only nonzero values are plotted). (a) Contangle $\tau_{\lambda \mid \nu}$ as a function of the mode frequencies, for a black hole of mass $M=1 /(2 \pi)$ and infinite entanglement $(\xi \rightarrow \infty)$ in the Kruskal frame. Inset: trade-off between frequencies and mass for $\xi \rightarrow \infty$; entanglement is nonzero only inside the convex meshed surface. (b) Contangle $\tau_{\lambda \mid \nu}$ (shaded surface) as a function of squeezing $\xi$ and mass $M$, for $\lambda=1$ and $\nu=2$, compared to the contangle $4 \xi^{2}$ (wireframe surface) detected by Kruskal observers.

mixed. We can exactly quantify entanglement in different partitions of (mixed) Gaussian states by using a measure known as the contangle $\tau$ [19. The contangle is equivalent to the Gaussian entanglement of formation [21, which quantifies the cost of creating a mixed Gaussian state out of an ensemble of pure entangled Gaussian states. In the pure-state case, the entanglement of formation reduces to the entropy of entanglement [16]. The entanglement between the modes $\lambda^{\text {out }}$ and $\nu^{\text {out }}$ from the perspective of observers outside the black hole is given by $\tau_{\lambda \mid \nu} \equiv \tau\left(\boldsymbol{\sigma}_{\lambda \nu}^{\text {out }}\right)=g\left[m_{\lambda \mid \nu}^{2}\right][19$, where

$$
m_{\lambda \mid \nu}=\frac{2 \mathrm{c}(2 l) \mathrm{c}(2 n) \mathrm{c}^{2}(\xi)+3 \mathrm{c}(2 \xi)-4 \mathrm{~s}(l) \mathrm{s}(n) \mathrm{s}(2 \xi)-1}{2\left[(\mathrm{c}(2 l)+\mathrm{c}(2 n)) \mathrm{c}^{2}(\xi)-2 \mathrm{~s}^{2}(\xi)+2 \mathrm{~s}(l) \mathrm{s}(n) \mathrm{s}(2 \xi)\right]}
$$

in case $\tanh (\xi)>\sinh (l) \sinh (n)$. Otherwise, the entanglement vanishes.

The bipartite entanglement depends on the degree of squeezing $\xi$ (which determines the entanglement described by Kruskal observers), on the frequencies $\lambda$ and $\nu$, and on the mass $M$ of the black hole. As shown in Fig. 1, entanglement increases with all these parameters. However, as function of the effective squeezing parameters $l$ and $n$ entanglement is strictly decreasing. We observe that the degradation on entanglement with decreasing mass is stronger if the entanglement $S_{\xi}$ in the Kruskal frame is larger. At fixed $\lambda$ and $\nu$, it is interesting to see that entanglement vanishes for a finite value of $M$. Conversely, note that for a fixed black hole mass $M$, the entanglement of low frequency modes vanishes or, better said, becomes physically unaccessible to the observers outside the black hole. Even in the ideal case, where the Kruskal state is an infinitely entangled EPR state [18] (corresponding to $\xi \rightarrow \infty$ ), the entanglement between modes $\lambda$ and $\nu$ completely vanishes if $e^{2 \pi \lambda M}+e^{2 \pi \nu M}-e^{2 \pi M(\lambda+\nu)} \geq 0$ [see inset of Fig. 1(a)]. Only modes with high frequency remain entangled. Moreover, the number of entangled modes becomes smaller for decreasing black hole mass and, as expected, the pairwise entanglement between two modes with given frequencies becomes weaker. In the limit $M \rightarrow 0$ the bipartite entanglement exactly vanishes between all frequency modes. The quantum correlations detected by Schwarzschild observers outside the black hole are irremediably lost, as we have just demonstrated in a full analytical fashion.

It is also possible to calculate the total correlations between the modes outside the black 


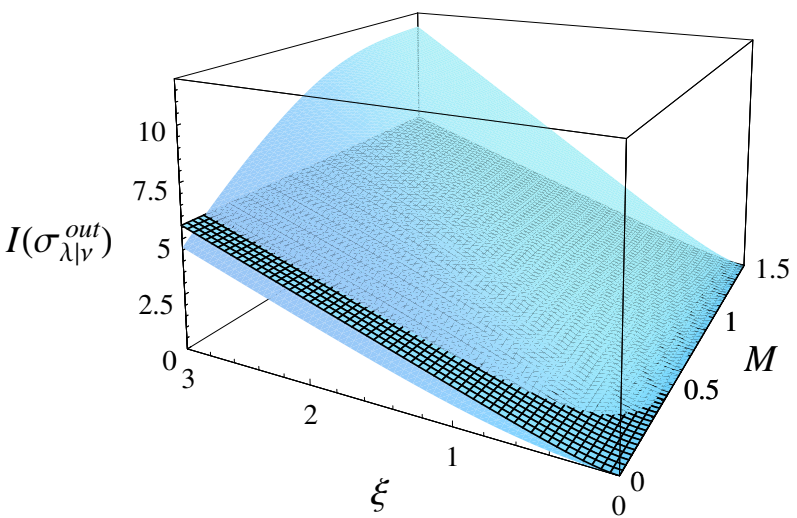

Fig. 2. Total correlations $I\left(\boldsymbol{\sigma}_{\lambda \nu}^{\text {out }}\right)$ (shaded surface) between modes with frequency $\lambda=1$ and $\nu=2$ detected outside a black hole, versus mass $M$ and squeezing $\xi$. The classical correlations $S_{\xi}$ detected by Kruskal observers are displayed as well (meshed surface).

hole using the mutual information [17] which in terms of the CM reads [22]

$$
I\left(\boldsymbol{\sigma}_{\lambda \nu}^{\text {out }}\right)=f\left(\sqrt{\operatorname{Det} \boldsymbol{\sigma}_{\lambda^{\text {out }}}}\right)+f\left(\sqrt{\operatorname{Det} \boldsymbol{\sigma}_{\nu^{\text {out }}}}\right)-f\left(\eta_{\lambda \nu}^{+}\right)-f\left(\eta_{\lambda \nu}^{-}\right),
$$

where $f(x)$ is defined in Eq. (3), and the symplectic eigenvalues are given by 23 .

$$
2\left(\eta_{\lambda \nu}^{ \pm}\right)^{2}=\Delta\left(\boldsymbol{\sigma}_{\lambda \nu}^{\text {out }}\right) \pm \sqrt{\Delta\left(\boldsymbol{\sigma}_{\lambda \nu}^{\text {out }}\right)^{2}-4 \operatorname{Det}\left(\boldsymbol{\sigma}_{\lambda \nu}^{\text {out }}\right)},
$$

with

$$
\begin{aligned}
\operatorname{Det}\left(\boldsymbol{\sigma}_{\lambda \nu}^{\text {out }}\right) & =\left[\mathrm{c}^{2}(n)+\mathrm{c}(2 \xi) \mathrm{s}^{2}(n)\right] \mathrm{c}^{2}(l)+\mathrm{s}^{2}(l)\left[\mathrm{c}(2 \xi) \mathrm{c}^{2}(n)+\mathrm{s}^{2}(n)\right]^{2}, \\
\Delta\left(\boldsymbol{\sigma}_{\lambda \nu}^{\text {out }}\right) & =\mathrm{c}^{2}(2 \xi) \mathrm{c}^{4}(l)+2\left[\mathrm{c}(2 \xi) \mathrm{s}^{2}(l)-\mathrm{c}^{2}(n) \mathrm{s}^{2}(2 \xi)\right] \mathrm{c}^{2}(l)+\mathrm{s}^{4}(l)+\left[\mathrm{c}(2 \xi) \mathrm{c}^{2}(n)+\mathrm{s}^{2}(n)\right]^{2} .
\end{aligned}
$$

The behavior of the mutual information is qualitatively similar to that of the bipartite entanglement. The most remarkable and original feature is that, in the regime where low frequency modes are disentangled (corresponding to a black hole with small mass, $M \rightarrow 0$ ), classical correlations are degraded as well. Namely (see Fig. 2), the mutual information $I\left(\boldsymbol{\sigma}_{\lambda \nu}^{\text {out }}\right)$ may fall below the threshold $S_{\xi}=f(\cosh 2 \xi)$, which corresponds to the classical correlations (and, equivalently, the entanglement) detected by Kruskal observers. Previous analyses reported classical correlations invariant under the Hawking effect, by considering either a maximally entangled two-qubit Kruskal state [14, or the correlations between modes measured by an observer falling into the black hole and the other escaping the fall [24, 25]. We find instead that, for a two-mode squeezed Kruskal state, classical correlations are strictly invariant only in the limit of infinite entanglement, $\xi \rightarrow \infty$, in which case for $M \rightarrow 0$, the total correlations $I\left(\boldsymbol{\sigma}_{\lambda \nu}^{\text {out }}\right) \rightarrow S_{\xi}$. For any finite degree of squeezing $\xi$ (which only then is physically meaningful), we have thus shown that Hawking radiation affects mostly quantum, but also classical correlations between modes outside a black hole. We observe that, nevertheless, the loss of classical correlations never exceeds one ebit.

\section{Genuine multipartite entanglement shared by inner and outer modes}

We are interested in understanding what happened to the lost quantum correlations. Are they really lost? Since the two entangled Kruskal modes are transformed into a four-partite 
system from the Schwarzschild perspective (with two modes, one inside and another outside the black hole, for each frequency $\lambda$ and $\nu$ ) we move to analyze the information stored in form of multipartite correlations in the state. Multipartite entanglement is a complex topic which can be addressed in very few cases, which fortunately include, thanks to some recent advances [20, 26, 27, Gaussian states. The measure of multipartite entanglement we use is called the residual contangle and stems from the observation that entanglement cannot be freely shared. There are 'monogamy' relations which impose trade-offs on the distribution of bipartite entanglement between the various mode partitions of the system [15], constraining the residual multipartite entanglement not stored in couplewise form. The minimum residual entanglement in the state $\boldsymbol{\sigma}_{\lambda \nu}^{\text {in,out }}$, derived from the entanglement monogamy inequality defined in [20, 26, 27, is given by the contangle between the mode $\lambda^{i n}$ and the rest, minus the contangle between $\lambda^{\text {in }}$ and $\lambda^{\text {out }}$, i.e., $\tau^{\text {res }}\left(\boldsymbol{\sigma}_{\lambda \nu}^{\text {in out }}\right)=\tau_{\lambda^{\text {in }} \mid\left(\lambda^{\text {out }} \nu^{\text {in }} \nu_{\text {out }}\right)}-\tau_{\lambda^{\text {in }} \mid \lambda^{\text {out }} \text {. Here we }}$. Hert labeled the modes such that $l \leq n$ without loss of generality, and used the fact that there are no quantum correlations between modes inside and outside of different frequency, nor between $\lambda^{i n}$ and $\nu^{\text {in }}$. Recalling that $\tau_{\lambda^{i n} \mid \lambda^{\text {out }}}=4 l^{2}$ and $\tau_{\nu^{\text {in }} \mid \nu^{\text {out }}}=4 n^{2}$, the residual contangle reads

$$
\tau^{r e s}=\operatorname{arcsinh}^{2}\left\{\sqrt{\left[\cosh ^{2} l+\cosh (2 \xi) \sinh ^{2} l\right]^{2}-1}\right\}-4 l^{2} .
$$

The derivation of this result is a straightforward generalization of the findings of Ref. 27, where the multipartite entanglement of the state in Eq. (4) has been studied, for $l=n$, in a quantum-optical setting. All technical details of these calculations are given in Refs. 27, 25].

This residual entanglement contains both tripartite and genuine four-partite contributions. The portion of tripartite correlations in the state, with respect to the above mode decomposition, only involves the mode $\lambda^{i n}$ and the two modes outside the black hole (the other contributions being zero). We calculate an upper bound to such tripartite entanglement between modes $\lambda^{\text {in }}, \lambda^{\text {out }}$ and $\nu^{\text {out }}$ using the techniques of Refs. [27, 25] and obtain,

$$
\tau^{t r i} \leq \min \left\{g\left[\left(\frac{\operatorname{sech}^{2}(n) \tanh ^{2}(\xi)+1}{\operatorname{sech}^{2}(n) \tanh ^{2}(\xi)-1}\right)^{2}\right]-g\left[m_{\lambda \mid \nu}^{2}\right], g\left[\left(\frac{\operatorname{sech}^{2}(n) \tanh ^{2}(\xi)-\cosh (2 l)}{\operatorname{sech}^{2}(n) \tanh ^{2}(\xi)-1}\right)^{2}\right]-4 l^{2}\right\} .
$$

The tripartite correlations vanish asymptotically for $l, n \gg 0$, which means that a portion of the information in the Kruskal state is redistributed into correlations with the remaining mode inside, $\nu^{i n}$, which are neither of bipartite nor of tripartite nature. Remarkably, in the regime of large $l, n$ (low frequencies and/or small black hole mass), we conclude that the residual entanglement given by Eq. (5) quantifies exclusively the genuine four-partite quantum correlations among all four modes.

We plot the residual entanglement in Fig. 3 where we see that it grows with the squeezing $\xi$. For fixed $\xi$, it increases with decreasing black hole mass $M$. Interestingly, the fourpartite entanglement can easily surpass the bipartite entanglement $4 \xi^{2}$ in the Kruskal state, and diverges in the limit $M \rightarrow 0$. Therefore, not only do we find that all the bipartite correlations in the Kruskal state are entirely redistributed into four-partite correlations among the Schwarzschild modes, but also that an unlimited amount of four-mode correlations is created by the presence of a black hole with infinitesimal mass. In this limit, the entanglement between modes of the same frequency across the horizon also diverges: this partly explains the generation of infinite genuine multipartite entanglement among all the four modes (the entanglement is said to distribute in a monogamous but 'promiscuous' way [27]) and why the 


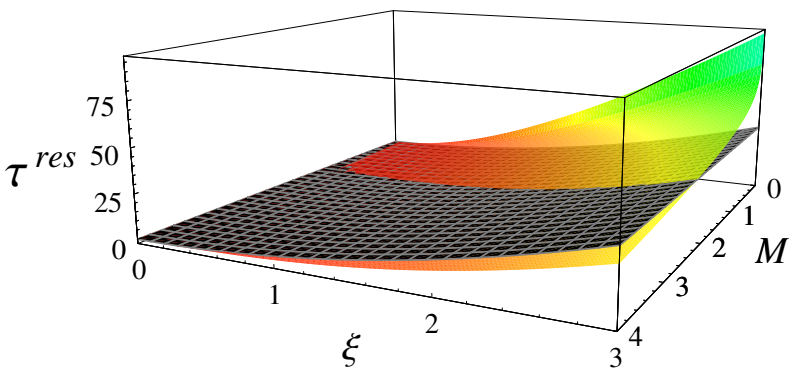

Fig. 3. Residual multipartite entanglement among the four (in and out) modes, Eq. (5) (shaded surface), versus squeezing $\xi$ and black hole mass $M$, at fixed $\lambda=1 /(8 \pi)$. The bipartite entanglement $4 \xi^{2}$ of the Kruskal state is displayed as well (meshed surface).

bipartite entanglement between the outside modes is degraded and, with decreasing mass, destroyed. The infinite energy, associated with such an infinite multipartite entanglement interlinking the inside and outside regions of the black hole horizon, is related to the infinite temperature at which the black hole radiates, according to the Hawking effect [7, in the small-mass limit 28 .

\section{Conclusions}

In this paper, we have characterized the nature of information loss in the case of entangled bosonic fields in the presence of a Schwarzschild black hole horizon. A novel, interesting signature of the Hawking mechanism [7] is derived: the detection of an even finite bipartite entanglement by infalling observers, amounts to the creation of an unbounded multipartite entanglement across the horizon. The limiting situation of a black hole with vanishing mass (a snapshot of its evaporation), commonly regarded as the origin of the information loss 'paradox' [6], is precisely identified by a divergence of such multipartite entanglement [28]. Already at finite black hole masses, observers outside the horizon may witness (depending on the mode frequencies) absolutely no entanglement in the field, and a small degradation of classical correlations as well. Connection of these insights to concepts of black hole thermodynamics awaits further investigation.

\section{Acknowledgments}

We thank F. Illuminati and M. Ericsson for discussions. I. F-S was supported by the Alexander von Humboldt Foundation and would like to thank Tobias Brandes for his hospitality.

\section{References}

1. M. M. Wolf, F. Verstraete, M. B. Hastings, and J. I. Cirac, Phys. Rev. Lett. 100, 070502 (2008).

2. M. A. Nielsen and I. L. Chuang, Quantum Computation and Quantum Information (Cambridge University Press, Cambridge, 2000).

3. J.A. Smolin and J. Oppenheim, Phys. Rev. Lett. 96, 081302 (2006); S. L. Braunstein and A. K. Pati, ibid. 98, 080502 (2007).

4. L. Bombelli, R. K. Koul, J. Lee, and R. D. Sorkin, Phys. Rev. D 34, 373 (1986); C. Callen and F. Wilzcek, Phys. Lett. B 333, 55 (1994).

5. H. Terashima, Phys. Rev. D 61, 104016 (2000).

6. For reviews see: J. Preskill, arXiv:hep-th/9209058 S. Carlip, arXiv:0705.3024. 
7. S. W. Hawking, Commun. Math. Phys. 43, 199 (1975); Phys. Rev. D 14, 2460 (1976); W. G. Unruh, ibid. 14, 870 (1976).

8. Kruskal (Schwarzschild) observers are defined by the vector field $\partial u(\partial t)$, where $u(t)$ is the Kruskal (Schwarzschild) temporal coordinate 9, 7]. A Kruskal (Schwarzschild) state/mode refers to the state/mode described by the respective observers.

9. N. D. Birrell and P. C. W. Davies, Quantum fields in curved space (Cambridge University Press, Cambridge, 1982).

10. D. Ahn, Phys. Rev. D 74, 084010 (2006).

11. J. Ball, I. Fuentes-Schuller, and F. P. Schuller, Phys. Lett. A 359, 550 (2006).

12. G. Adesso and F. Illuminati, J. Phys. A 40, 7821 (2007).

13. In this respect we notice that the degradation of correlations in a different type of two-mode entangled Kruskal state, i.e. a two-qubit-like Bell state $|00\rangle+|11\rangle$, as detected by Schwarzschild observers, have been recently studied 14]. However, in that case it was not possible to frame the effect of black hole within the theory of entanglement redistribution, nor a degradation of classical correlations was observable.

14. D. Ahn, J. Korean Phys. Soc. 50368 (2007).

15. B. M. Terhal, IBM J. Res. \& Dev. 48, 71 (2004); G. Adesso and F. Illuminati, Int. J. Quant. Inf 4, 383 (2006).

16. The entropy of entanglement of a bipartite state $|\psi\rangle_{A B}$ is defined as $S\left(\varrho_{A}\right)=S\left(\varrho_{B}\right)=$ $-\operatorname{tr}\left[\varrho_{A} \log \left(\varrho_{A}\right)\right]$, where $\varrho_{A}\left(\varrho_{B}\right)$ is the reduced density matrix of subsystem $A(B)$.

17. B. Groisman, S. Popescu, and A. Winter, Phys Rev A 72, 032317 (2005).

18. A. Einstein,B. Podolsky, and N. Rosen, Phys. Rev. 47, 777 (1935).

19. The contangle is defined as $\tau\left(\boldsymbol{\sigma}_{i \mid j}\right) \equiv g\left[m_{i \mid j}^{2}\right]$ where $g[x]=\operatorname{arcsinh}^{2}[\sqrt{x-1}]$ and $\boldsymbol{\sigma}_{i \mid j}$ is the CM of a (mixed) bipartite Gaussian state where subsystem $i$ comprises one mode only 20]. Here $m_{i \mid j} \equiv m\left(\boldsymbol{\sigma}_{i \mid j}^{o p t}\right)=\left(\operatorname{Det} \boldsymbol{\sigma}_{i}^{o p t}\right)^{1 / 2}$, where $\boldsymbol{\sigma}_{i}^{o p t}$ is the reduced CM of subsystem $i$. The CM $\boldsymbol{\sigma}_{i \mid j}^{o p t}$ denotes the pure bipartite Gaussian state which minimizes $m\left(\boldsymbol{\sigma}_{i \mid j}^{p}\right)$ among all pure-state CMs $\boldsymbol{\sigma}_{i \mid j}^{p} \leq \boldsymbol{\sigma}_{i \mid j}$. Separability amounts to $m_{i \mid j}=1$.

20. G. Adesso and F. Illuminati, New J. Phys. 8, 15 (2006).

21. M. M. Wolf, G. Giedke, O. Krüger, R. F. Werner, and J. I. Cirac, Phys. Rev. A 69, 052320 (2004); G. Adesso and F. Illuminati, ibid. 72, 032334 (2005)

22. A. Serafini, F. Illuminati, and S. De Siena, J. Phys. B 37, L21 (2004).

23. G. Adesso, A. Serafini, and F. Illuminati, Phys. Rev. Lett. 92, 087901 (2004); Phys. Rev. A 70, 022318 (2004).

24. I. Fuentes-Schuller and R. B. Mann, Phys. Rev. Lett. 95, 120404 (2005); P. M. Alsing, I. FuentesSchuller, R. B. Mann, and T. E. Tessier, Phys. Rev. A 74, 032326 (2006); D. Ahn and M. S. Kim, Phys. Lett. A 366, 202 (2007).

25. G. Adesso, I. Fuentes-Schuller and M. Ericsson, Phys. Rev. A 76, 062112 (2007).

26. T. Hiroshima, G. Adesso, and F. Illuminati, Phys. Rev. Lett. 98, 050503 (2007).

27. G. Adesso, M. Ericsson, and F. Illuminati, Phys. Rev. A 76, 022315 (1007).

28. An infinitely entangled state, accompanied by unbounded energy, is unphysical. The appearance of such states is a well known issue in black hole physics, and only a satisfactory theory of quantum gravity might be able to "renormalize" these divergences by properly describing the physics of a black hole close to evaporation. In fact, Hawking's semiclassical calculations [7] within the formalism of quantum field theory in curved spacetimes [9], break down at the Planck scale. We can then safely conclude that our results concerning correlation loss and entanglement redistribution are physical at least for black hole masses $M$ above the Planck mass $M_{p}=1$ (in natural units). This corresponds to introducing a cutoff on the radiated energy and thus on the distributed entanglement. Quantitatively, from Fig. 3 and Eq. (5) one can see that, e.g. setting $\lambda=1 /(8 \pi)$, the four-partite entanglement at the Planck scale is $\tau^{\text {res }} \approx 20$ ebits for $\xi=2, \tau^{r e s} \approx 45$ ebits for $\xi=3$, and so on. It increases with increasing squeezing $\xi$ and decreasing frequency $\lambda$. 